\title{
Freely suspended two-dimensional electron gases
}

\author{
R.H. Blick ${ }^{\mathrm{a}, *}$, M.L. Roukes ${ }^{\mathrm{a}}$, W. Wegscheider ${ }^{\mathrm{b}}$, M. Bichler ${ }^{\mathrm{b}}$ \\ ${ }^{a}$ California Institute of Technology, Condensed Matter Physics 114-36, Pasadena, CA 91125, USA \\ ${ }^{\mathrm{b}}$ Walter Schottky Institut der TU München, Am Coulombwall, 85748 Garching, Germany
}

\begin{abstract}
We present a new technique allowing us to build freely suspended two-dimensional electron gases from AlGaAs/GaAs/AlAs heterostructures. This technique relies on an MBE-grown structures that includes a sacrificial layer. (C) 1998 Elsevier Science B.V. All rights reserved.
\end{abstract}

Keywords: $\mathrm{AlGaAs} / \mathrm{GaAs} / \mathrm{AlAs}$; Heterostructures

The overall aim of this work is to provide new approaches for studying the properties of nanomachined structures. Recently, the realization of suspended, monocrystalline, GaAs nanostructures containing a three-dimensional electron gas has been demonstrated [1]. In other work, the possibility of using a GaAs HEMT to achieve sensitive piezoelectric detection of strain has been shown [2]. We are combining these techniques to enable a new means of detecting motion in nanostructures. These structures should also enable unique studies of phonon and photon interactions in 2DEG devices that are strongly decoupled from the substrate.

Sensitive displacement detectors with subnanometer resolution and bandwidths into the microwave range are required for emerging applications with nanometer scale electromechanical devices [3]. The high mobility 2DEG system pro-

\footnotetext{
* Corresponding author. Fax: 1 (626)683 9060; e-mail: blick@ cco.caltech.edu.
}

vides a unique approach to implementing such wideband, extremely sensitive displacement detection, through use of the piezoelectric properties of GaAs to modulate a suspended nanometer-scale HEMT structure.

To create such devices, we employ new processing techniques involving specially designed, MBEgrown, 2DEG heterostructures which include a sacrificial layer. Two different types of sacrificial layers for the processing, formed from either AlAs or $\mathrm{Al}_{0.8} \mathrm{Ga}_{0.2} \mathrm{As}$, have been investigated. A GaAs layer in the center of the heterostructure forms a well-defined conduction channel with a typical width of approximately $15 \mathrm{~nm}$. The entire suspended layer, which includes the Si dopant layers and the bottom and top cap layers, has a thickness of about $100 \mathrm{~nm}$. The AlAs sacrificial layer thickness is of order $400 \mathrm{~nm}$; this layer is removed by a HF wet etch. The two-dimensional electron gas (2DEG) used in this experiment is located $40 \mathrm{~nm}$ below the sample surface. The carrier density of the 

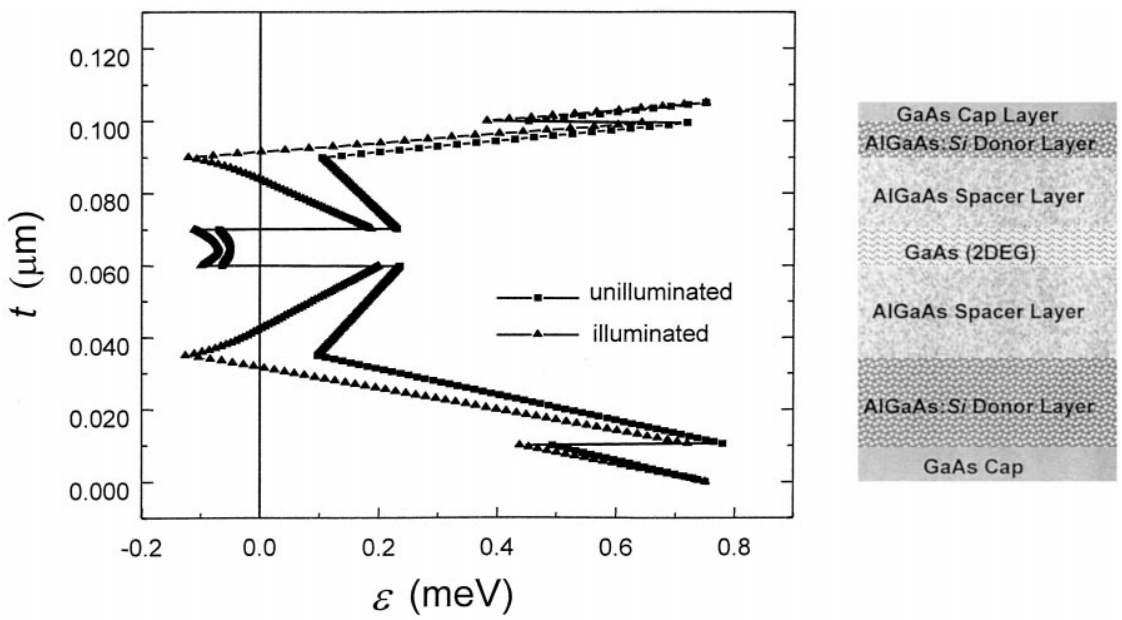

Fig. 1. The processing steps to suspend the two-dimensional electron gas: (a) definition of the optical mask with antenna structure; (b) preparation of ohmic contacts and the Ni etch mask; (c) ion beam etch defines a mesa; (d) the final wet etch removes the sacrificial layer to yield the freely suspended electron gas.
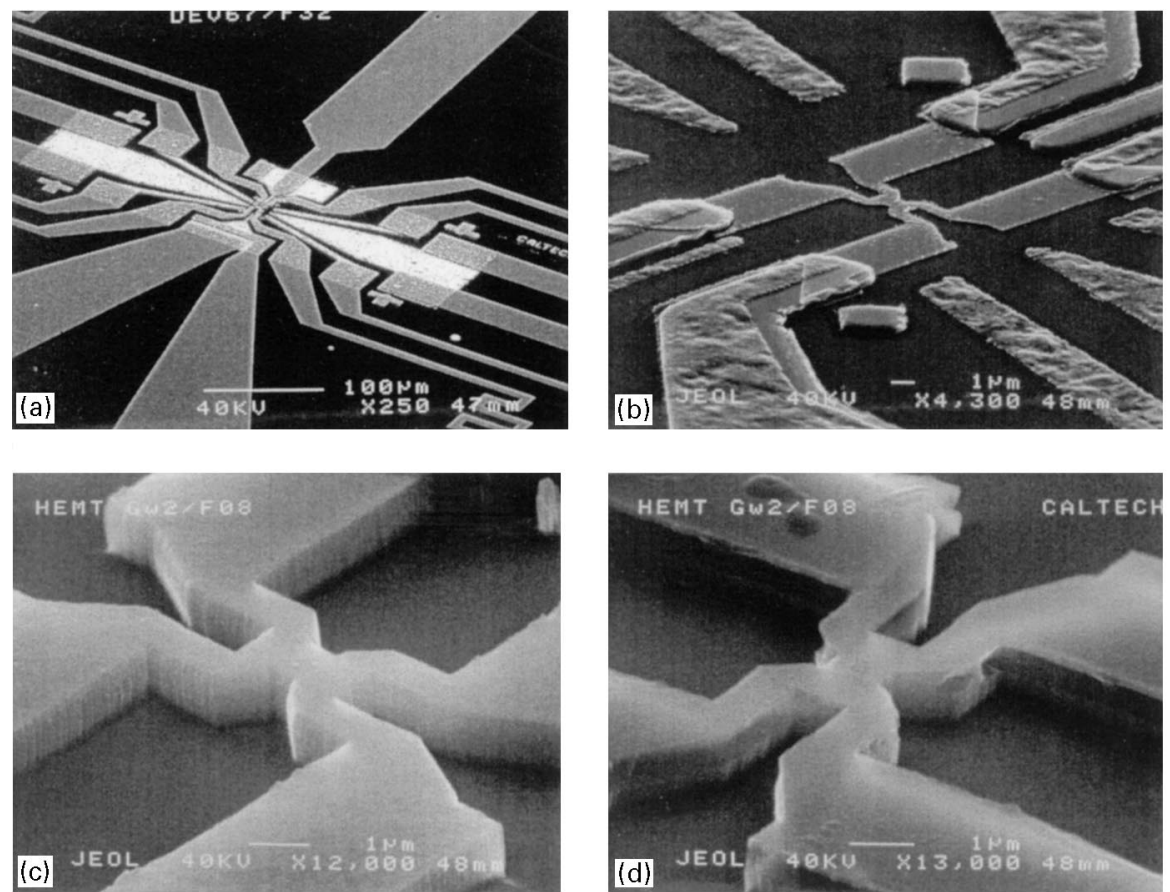

Fig. 2. Calculation of the band structure of the freely suspended two-dimensional electron gas. As indicated, the filled squares mark the unilluminated trace and the triangles the illuminated one. The Fermi level is pinned at mid-gap for the exposed top and bottom surfaces. The temperature assumed in the calculation is $T=10 \mathrm{~K}$.

unsuspended $2 \mathrm{DEG}$ at a temperature of $4.2 \mathrm{~K}$ is $2.64 \times 10^{15} \mathrm{~m}^{-2}$ with a mobility of $51 \mathrm{~m}^{2} / \mathrm{V} \mathrm{s}$ in the dark. The conduction band lineup for these sam- ples, as calculated by Frank Stern [4] is shown in Fig. 1. The calculations use the Poisson-Schrödinger solver program SCRAPS, written by Steven E. 
Laux and Arvind Kumar, which neglects manybody effects [5].

Optical and electron beam lithography, followed by a combination of anisotropic ion beam (dry) and chemically selective (wet) etching techniques are applied to realize suspended nanostructures with three-dimensional relief. The processing procedure is depicted in Fig. 2: the first step is the fabrication of ohmic contacts, then a Hall-bar structure $(a, b)$ is defined lithographically and then etched by ion beam assisted etching into the substrate. The vertically etched structure is shown in Fig. 2c. The subsequent, final, step is removal of the sacrificial layer using a chemically selective etch. The resulting freely suspended 2DEG is shown in Fig. 2d.

Special care was taken in the optical mask layouts, since the coupling of microwaves is crucial for our experiments. To simulate this we employed a commercially available computer program [6]. In Fig. 3, calculations of the coupling of $4 \mathrm{GHz}$ microwaves to the metal gates on top of the GaAs hetero- structure are shown. The shading on the metal gates represents the intensity of the high-frequency current density. White corresponds to a high density; dark grey represents low current density. As seen in Fig. 3a, the antenna is driven by the excitation on port \#5, which leads to an oscillating field across the center of the device. In Fig. $3 b$ the 2 DEG layer is shown, as expected we find a high field intensity at the center device under test.

The significance of freely suspended structures that include a high-mobility electron gas lies in their versatility. They can be applied to create new devices, e.g. sensitive bolometers, as well as model systems for heat capacity measurements on 2DEGs [7]. The sensitivity of a nanometer-scale bolometer is enhanced by its small volume which is on the order of $\sim 3 \mu^{3}$, as compared to usual dimensions of $\sim 10^{8} \mu \mathrm{m}^{3}$. The low shunting capacitance due to the lack of bulk material beneath the active area may also yield highly efficient photon detection.
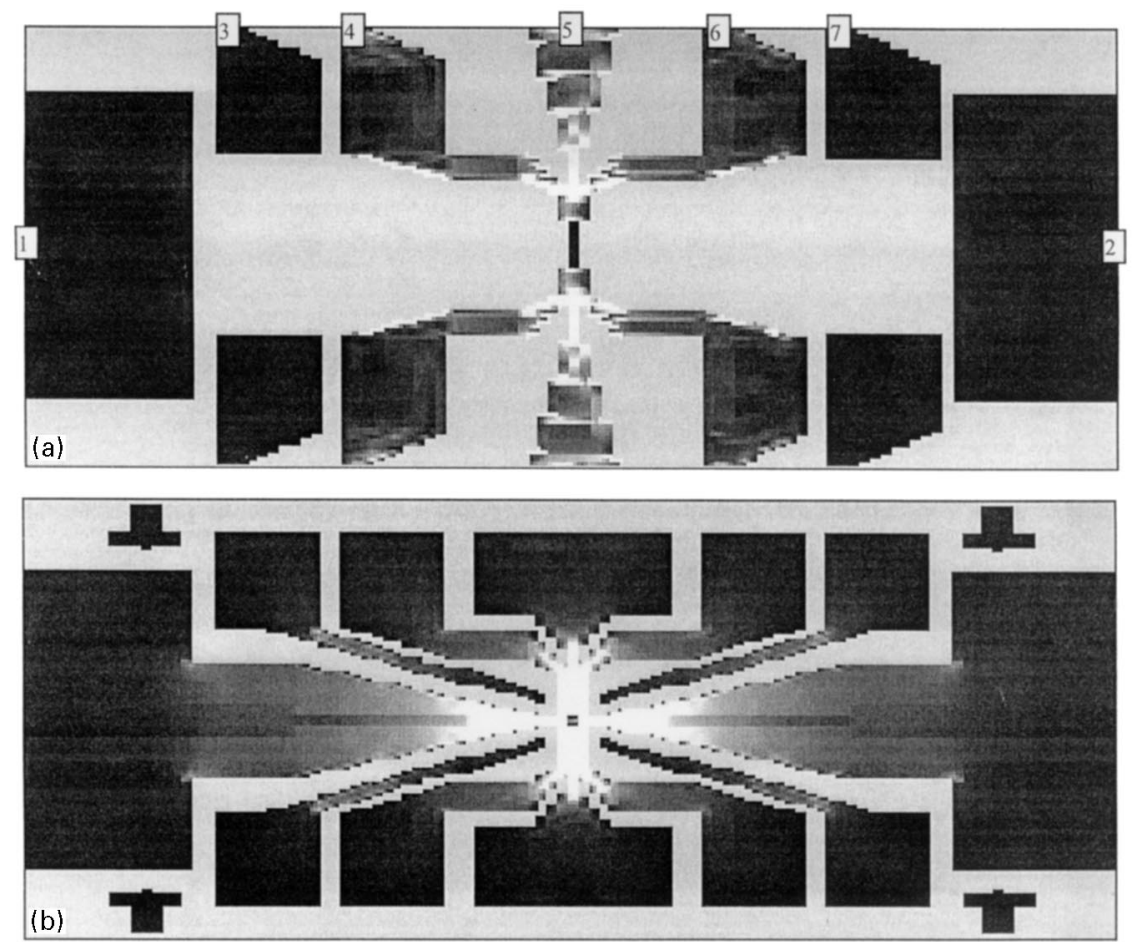

Fig. 3. Calculated coupling of $4 \mathrm{GHz}$ microwaves tothe metal gates on top of the GaAs heterostructures: (a) shows the device as a whole; (b) the 2DEG lines feeding the device under test (see text for details). 
We gratefully acknowledge the help of Frank Stern in modeling the heterostructure. We also thank Andrew Cleland and Frank Monzon for technical assistance and lively discussions. R.H.B. thanks the Max Planck Gesellschaft (Otto-Hahn stipend) and the Alexander-von-Hunmboldt Stiftung (Lynen stipend) for support. This work is supported by DARPA, under Grant DABT63-95C-0112.

\section{References}

[1] T.S. Tighe, J.M. Worlock, M.L. Roukes, Appl. Phys. Lett. 70 (1997) 2687.

[2] R.G. Beck et al., Appl. Phys. Lett. 68 (1996) 3763.

[3] H. Blauvelt et al., Appl. Phys. Lett. 40 (1982) 289; A.N. Cleland, M.L. Roukes, Appl. Phys. Lett. 69 (1996) 2653.

[4] F. Stern, personal communication.

[5] A. Kumar, S.E. Laux, F. Stern, Phys. Rev. B 42 (1990) 5166.

[6] Sonnet Software, version 4.0, Liverpool, NY (1997).

[7] J.K. Wang et al., Phys. Rev. B 38 (1988) 6174. 